\title{
Fabricating a Prototype Spectrometer Using a Large-Angle Direct UV-Written Chirped Tilted Grating
}

\author{
James W. Field, Matthew T. Posner, Sam A. Berry, Rex H. S. Bannerman, James C. Gates, Peter G.R. Smith \\ Optoelectronics Research Centre, Building 46, University of Southampton, Highfield Campus, Hampshire, SO17 1BJ \\ j.field@soton.ac.uk
}

\begin{abstract}
A prototype planar waveguide spectrometer is fabricated with a $45^{\circ}$ direct $\mathrm{UV}$-written grating and a chirp of $140 \mathrm{~nm}$. The grating focus translates by $1-2 \mu \mathrm{m} / \mathrm{nm}$ of input wavelength tuning, over a bandwidth of more than $200 \mathrm{~nm}$.
\end{abstract}

\section{Introduction}

Many integrated optics sensing systems rely on detecting changes in a spectrum based on varying external conditions. Integrated optics provides a route to cheap mass-producible sensors; however typically require expensive and bulky devices to analyze such changes. Using the reflection properties of tilted gratings in planar waveguides, it is possible to create a device that focusses light out of a waveguide, with focal position dependent on wavelength. Small angle devices have been previously demonstrated [1,2], however large angle devices such as the one reported here show a much increased bandwidth. They are also expected to have a far greater outcoupling efficiency and should only operate on one polarization mode, reducing noise from birefringence.

In a tilted grating the observation angle of light reflected (relative to the waveguide direction) is given by [3]:

$$
\frac{\lambda \cos \theta_{T}}{n_{e f f} \Lambda(\mathrm{z})}-1=\cos \theta_{o},
$$

where $\lambda$ is the input wavelength, $\theta_{T}$ is the angle between the normal to the grating plane and the direction of propagation, $n_{\text {eff }}$ is the waveguide effective index, $\Lambda(\mathrm{z})$ is the grating period at position $\mathrm{z}$ and $\theta_{o}$ is the angle between the direction of propagation and the observation angle of the light. By varying the grating period along the length of the grating, i.e. introducing chirp, the light reflected from different points along the tilted grating converges at a focus.

Direct UV writing (DUW)[3], is a laser writing method used to simultaneously define waveguides and gratings in photosensitive silica glass. The fabrication technique permits low loss integration into fiber networks through well-matched waveguide numerical apertures with commercial fiber technologies. By combining computercontrolled grating chirp [3] with large angle fabrication tools [4] it is possible to create small footprint low-cost planar spectrometers.

\section{Method}

A waveguide was fabricated with a $1 \mathrm{~mm}$ linearly chirped tilted grating in a silica-on-silicon planar chip via DUW. The grating has a tilt angle of 45 degrees, with a linear period chirp of $140 \mathrm{~nm}$ over the grating, centered at a design wavelength of $1550 \mathrm{~nm}$.

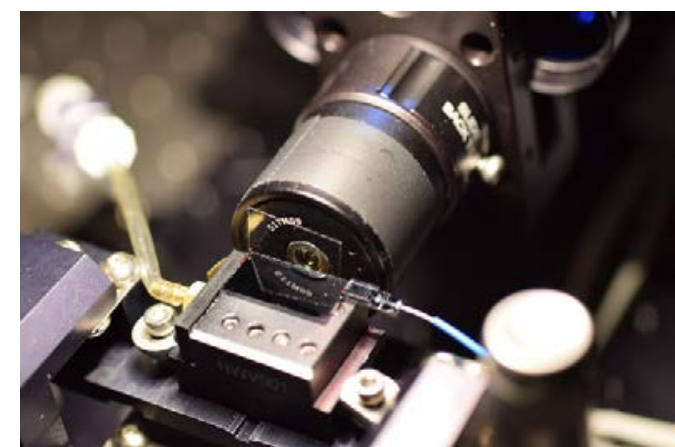

Fig. 1. Fabricated device pigtailed with camera for interrogation. Chip length and width are $20 \mathrm{~mm}$ and $10 \mathrm{~mm}$ repsectively

A $1550 \mathrm{~nm}$ PM pigtail was used to couple into the device (Fig.1), and a $1550 \mathrm{~nm}$ linear camera (Raptor photonics OW1.7-VS-CL-640) was used to image the focus of the grating in the near-infrared. A $200 \mu \mathrm{m}$ coverslip and 
refractive index matching oil $(\mathrm{n}=1.44)$ were used to reduce aberrations from an unpolished facet. A narrow linewidth polarized tunable laser (Agilent 8164B) was used to investigate the bandwidth, wavelength sensitivity and spot size.

\section{Results and Discussion}

The output of the camera (Fig 2.) shows the output of the device moving as the wavelength of the input light is changed. The device was tested over the entire range of the tunable source (1440-1640 nm) and saw no noticeable change in spot size or shape. From the camera data the central spot has a width of approximately $8 \mu \mathrm{m}$, and moves by 1-2 $\mu \mathrm{m}$ per nm of wavelength tuning. A number of lobes can be seen on the right hand side of the focal spot, these are attributed to the grating apodization. Due to the magnification of the camera setup the linearity of the movement of the central spot could only be confirmed between 1530 and $1570 \mathrm{~nm}$ before the spot moved outside of the detector area.
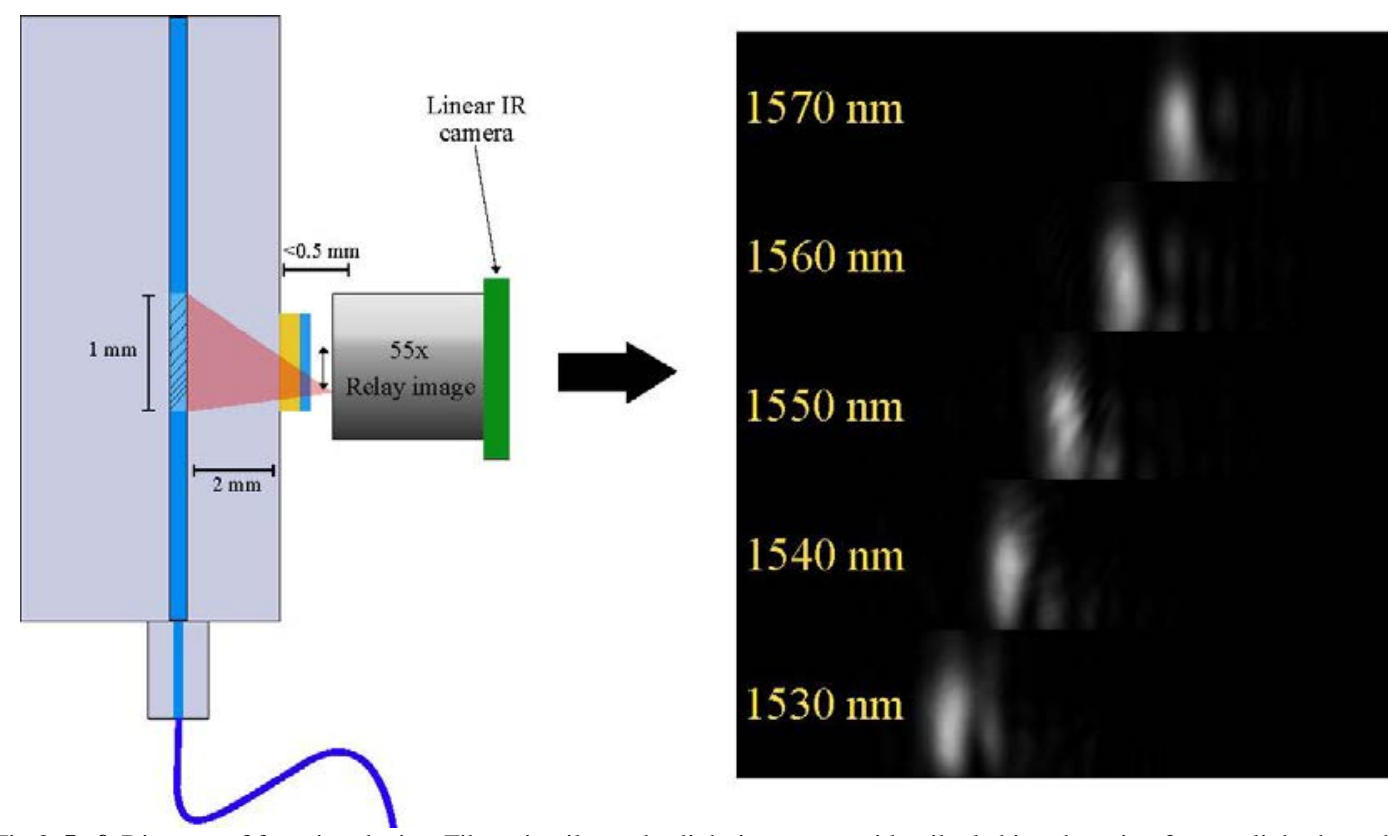

Fig 2. Left Diagram of focusing device. Fiber pigtail couples light into waveguide, tilted chirped grating focuses light through index matching oil (yellow) and cover slip (light blue) onto camera. Right Camera image of focal spot for different wavelengths. The position of the camera is fixed across all images.

\section{Future Perspectives}

The device presented here is an initial proof of concept and has much scope for optimization. A successor will aim to reduce the focal spot size and change the shape by apodizing the grating and improving the chirp function. The device will be characterized in more depth, including its polarization sensitivity.

\section{References}

[1] C.K.Madsen, J. Wagener, T.A. Strasser, D. Muehlner, M.A. Milbrodt, E.J. Laskowski and J. DeMarco, "Planar Waveguide Optical Spectrum Analyzer Using a UV-Induced Grating,” IEEE J. Quantum Electron. 4(6) 925-929 (1998)

[2] C. Koeppen, J.L. Wagener, T.A. Strasser and J. J. DeMarco , “High Resolution Fiber Grating Optical Network Monitor,” NFOEC Proceedings, Session 17 p13-19 (1998)

[3] C. Sima, J.C. Gates, H.L. Rogers, P.L. Mennea, C. Holmes, M.N. Zervas and P.G.R. Smith, "Ultra-wide detuning planar Bragg grating fabrication technique based on direct UV grating writing with electro-optic phase modulation,” Opt. Express 21(13) 15747-15754 (2013)

[4] M.T. Posner, P.L. Mennea, N. Podoliak, P. Horak, J.C. Gates and P.G.R. Smith “45 tilted gratings for silica-based integrated polarizers," CLEO Proceedings, CE_10_5 (2015) 